\title{
Seasonal congestive heart failure mortality and hospitalisation trends, Quebec 1990-1998
}

\section{Ehrmann Feldman, R Platt, V Déry, C Kapetanakis, D Lamontagne, A Ducharme, N Giannetti, M Frenette, E J Beck}

\begin{abstract}
Study objective: To describe seasonal congestive heart failure (CHF) mortality and hospitalisations in Quebec, Canada between 1990-1998 and compare trends in CHF mortality and morbidity with those in France.

Design: Population cohort study.

Setting: Province of Quebec, Canada.

Patients: Mortality data were obtained from the Quebec Death Certificate Registry and hospitalisation from the Quebec Med-Echo hospital discharge database. Cases with primary ICD-9 code 428 were considered cases of CHF.

Results: Monthly CHF mortality was higher in January, declined until September and then rose steadily $(p<0.05)$. Hospital admissions for CHF declined from May until September (moving averages analysis $\mathrm{p}<0.0001$ ). Seasonal mortality patterns observed in Quebec were similar to those observed in France.

Conclusion: CHF mortality in Quebec is highest during the winter and declines in the summer, similar to observations in France and Scotland. This suggests that absolute temperatures may not necessarily be that important but increased CHF mortality is observed once environmental temperatures fall below a certain "threshold" temperature. Alternatively better internal heating and warmer clothing required for survival in Quebec may ameliorate mortality patterns despite colder external environments.
\end{abstract}

$\mathrm{S}$ tudies have shown an association between higher congestive heart failure (CHF) mortality ${ }^{1}$ and hospital admissions $^{12}$ during the colder seasons. In Quebec, seasonal variability in environmental temperatures is considerable and it would be expected that CHF mortality rates would be higher in winter compared with regions with more temperate climates. The objectives of this study were to describe seasonal CHF mortality and hospitalisation rates between 1990 and 1998 and to compare these patterns with those in France.

\section{METHODS}

Mortality and hospitalisation data for the years 1990 to 1998 were obtained from Quebec population administrative databases (death certificate registry and hospital discharge databases). All persons with a primary diagnosis of ICD-9 code 428 comprised cases of CHF.

Seasonal averages were calculated for each of the threemonthly periods across the study years 1990-1998. Monthly averages were calculated across the study years. We used ARIMA (autoregressive integrated moving average) models to test for seasonal trends. We compared CHF mortality and hospitalisations in Quebec with those in France $^{1}$ by computing percentages above or below the average monthly

\section{Key points}

- Mortality and morbidity for heart failure were higher in the winter than in summer.

- Seasonal trends in CHF mortality were similar for Quebec and France.

- Winters are significantly colder in Quebec, suggesting that increased mortality is observed once temperatures fall below a certain "threshold".

value for the study period. SAS version 6.12 was used for all analyses.

\section{RESULTS}

Monthly CHF mortality was highest in January, decreasing until September and then rising again. It ranged from a high of $9.7 \%$ of all CHF deaths in January to a low of $7.3 \%$ in September. Both moving averages trend analysis $(p<0.0048)$ and autoregressive components $(\mathrm{p}<0.0001)$ demonstrated significant seasonal trends for mortality. Hospital admissions for CHF declined from May until September and ranged from a high of $9.2 \%$ in March to a low of $7.1 \%$ in August. Hospitalisation showed a statistically significant seasonal trend for the 12 month moving average analysis $(\mathrm{p}<0.0001)$, but not the autoregressive component (fig 1).

CHF mortality in Quebec followed a similar pattern to that observed in France. January was the month with the highest CHF mortality: $25 \%$ above the monthly average in Quebec and $20 \%$ above the monthly average in France. The month with the lowest mortality in Quebec was September, 16\% below the monthly average, while in France August was 15\% below average. These trends did not differ statistically $(\mathrm{p}=0.3500)$.

The range of monthly differences in hospitalisations in Quebec differed by $12.5 \%$, from $6.7 \%$ above the monthly average of admissions in January, to $5.8 \%$ below the monthly average of admissions in July. The corresponding highest and lowest monthly values in France ranged across 30\%, from $10 \%$ above the monthly average hospitalisations in April to $20 \%$ below this average in August. Differences in hospitalisation rates between Quebec and France were significant $(\mathrm{p}<0.0001)$.

\section{DISCUSSION}

Cold weather, among other factors, increases peripheral vasoconstriction, which may lead to pulmonary oedema as a consequence of left ventricular failure and may also cause abnormal clotting. ${ }^{3}$ In patients with symptomatic CHF, cold exposure decreases exercise capacity and increases the already raised systemic adrenergic activation. ${ }^{4}$ As increased 

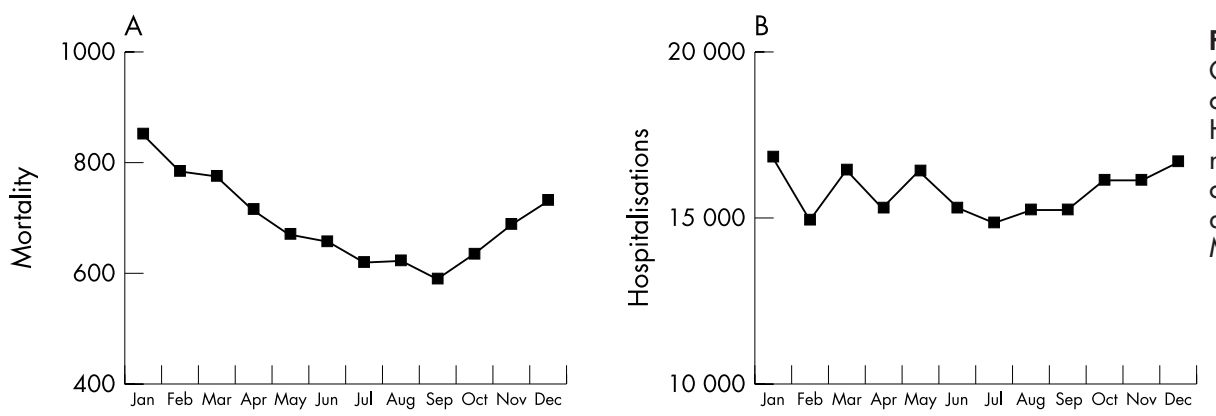

Figure 1 (A) Mortality from CHF in Quebec - number of deaths per month over the eight year period. (B) Hospitalisations from CHF in Quebecnumber of hospitalisations per month over the eight year period. (C) The high and low temperatures per month, in Montreal, the province's largest city.

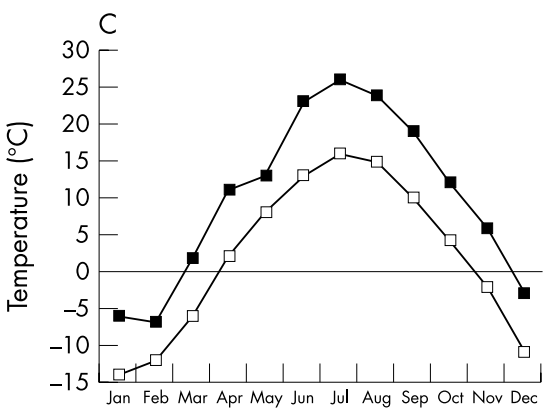

\section{Policy implications}

- Preventive measures should be developed and implemented to anticipate seasonal variations.

- Monitoring of CHF service provision and outcomes is important if we are to understand this disease and provide solutions for improvement of service provision.

plasma norepinephrine concentrations are associated with increased risk of death from progressive heart failure ${ }^{5}$ this may, in part, explain the increased mortality in winter.

Lower summer admissions may be attributable to bed closures during the summer-a common practice in many Quebec hospitals. In France, August is the month of summer vacation; closure of wards, cancellation of clinics and people travelling out of the country, may all contribute to lower hospital admissions. Conversely, because of the adverse environmental temperatures in winter, patients may be admitted more readily during this season as it may be more difficult to travel to and fro for outpatient appointments.

Comparisons between Quebec and France indicate similar trends with respect to seasonality of mortality. A Scottish study showed similar trends as well. ${ }^{2}$ These similarities occurred despite the fact that temperatures are considerably lower in Quebec than in France during winter and are more similar in summer. This may suggest that absolute temperatures may not be that important but increased deaths from CHF may occur when temperatures fall below a certain "threshold" level. Alternatively better internal heating and warmer clothing required for survival in Quebec may ameliorate mortality patterns despite colder external environments. Preventive measures should be developed to anticipate seasonal variations.

\section{ACKNOWLEDGEMENTS}

Direction de la Santé Publique de Montréal Centre. Dr Ehrmann Feldman is the recipient of a new investigator award from the Canadian Institutes of Health Research.

\section{Authors' affiliations}

D Ehrmann Feldman, V Déry, C Kapetanakis, D Lamontagne, E J Beck, Direction de la Santé Publique de Montréal Centre, Canada

D Ehrmann Feldman, Université de Montréal, GRIS et École de réadaptation, Canada

R Platt, E J Beck, McGill University, Joint Department of Epidemiology, Biostatistics and Occupational Health, Montreal, Canada

V Déry, Département de médecine sociale et préventive, Université de Montreal and Institut national de santé publique du Quebec, Canada

A Ducharme, Institut de cardiologie de Montreal, Canada

N Giannetti, McGill University Health Centre, Royal Victoria Hospital, Montreal, Canada

M Frenette, Hôpital Sacre-Coeur, Montreal, Canada

Correspondence to: Dr D Ehrmann Feldman, Direction de la Santé Publique de Montréal Centre, 1301 Sherbrooke est, Montreal, Quebec H2L 1M3, Canada; debbie.feldman@umontreal.ca

Accepted for publication 15 October 2003

\section{REFERENCES}

1 Boulay F, Berthier F, Sisteron O, et al. Seasonal variation in chronic heart failure hospitalizations and mortality in France. Circulation 1999;100:280-6.

2 Stewart S, Mclntyre K, Capewell S, et al. Heart failure in a cold climate: seasonal variation in heart failure-related morbidity and mortality. J Am Coll Cardiol 2002;39:760-6.

3 Wilmshurst P. Temperature and cardiovascular mortality. BMJ 1994:309:1029-30.

4 White $M$, Blanchet $M$, Ducharme A, et al. Beta-adrenergic blockers attenuate exercise-induced adrenergic activation and cold-induced impairment in exercise performance in patients with congestive heart failure. J Am Coll Cardiol 2001;37(suppl A):281A.

5 Cohn JN, Levine TB, Olivari MT, et al. Plasma norepinephrine as a guide to prognosis in patients with chronic congestive heart failure. N Engl J Med 1984;311:819-23. 